\title{
Structural mimicry confers robustness in the cyanobacterial circadian clock
}

Joel Heisler ${ }^{1,2} \uparrow$, Jeffrey A. Swan ${ }^{3} \dagger$, Joseph G. Palacios ${ }^{3}$, Cigdem Sancar ${ }^{4}$, Dustin C. Ernst ${ }^{4}$,

Rebecca K. Spangler ${ }^{3}$, Clive R. Bagshaw ${ }^{3}$, Sarvind Tripathi ${ }^{3}$, Priya Crosby ${ }^{3}$, Susan S. Golden ${ }^{4,5}$,

Carrie L. Partch ${ }^{3,5 *}$, Andy LiWang $1,2,5,6,7,8,9 *$

${ }^{1}$ Graduate Program in Chemistry and Chemical Biology, University of California, Merced, CA 95343.

${ }^{2}$ Center for Cellular and Biomolecular Machines, University of California, Merced, CA 95343.

${ }^{3}$ Department of Chemistry \& Biochemistry, University of California, Santa Cruz, CA 95064.

${ }^{4}$ Division of Biological Sciences, University of California, San Diego, La Jolla, CA 92093.

${ }^{5}$ Center for Circadian Biology, University of California, San Diego, La Jolla, CA 92093.

${ }^{6}$ School of Natural Sciences, University of California, Merced, CA 95343.

${ }^{7}$ Quantitative \& Systems Biology, University of California, Merced, CA 95343.

${ }^{8}$ Center for Cellular and Biomolecular Machines, University of California, Merced, CA 95343.

${ }^{9}$ Health Sciences Research Institute, University of California, Merced, CA 95343.

†These authors contributed equally to this work

*Correspondence should be addressed to A.L. (aliwang@ucmerced.edu) or C.L.P. (cpartch@ucsc.edu).

\section{Short title:}

SasA-KaiB mimicry and circadian rhythms 


\begin{abstract}
:
The histidine kinase SasA enhances robustness of circadian rhythms in the cyanobacterium $S$. elongatus by temporally controlling expression of the core clock components, kaiB and kaiC. Here we show that SasA also engages directly with $\mathrm{KaiB}$ and KaiC proteins to regulate the period and enhance robustness of the reconstituted circadian oscillator in vitro, particularly under limiting concentrations of KaiB. In contrast to its role regulating gene expression, oscillator function does not require SasA kinase activity; rather, SasA uses structural mimicry to cooperatively recruit the rare, fold-switched conformation of KaiB to the KaiC hexamer to form the nighttime repressive complex. Cooperativity gives way to competition with increasing concentrations of SasA to define a dynamic window by which SasA directly modulates clock robustness.
\end{abstract}

\title{
One Sentence Summary:
}

SasA controls the assembly of clock protein complexes through a balance of cooperative and competitive interactions. 


\section{Main Text:}

The core circadian clock genes kaiA, kaiB, and kaiC, are essential for rhythmic gene expression in cyanobacteria (1) and their proteins generate a $\sim 24$-hour rhythm of phosphorylation of KaiC in vivo (2) that persists in the absence of transcription or translation (3). Reconstitution of this posttranslational oscillator (PTO) in vitro (4) has led to deep insight into the structural and kinetic mechanisms that regulate formation of the clock protein assemblies and underlie cyanobacterial circadian rhythms. KaiC autophosphorylation is stimulated by KaiA during the day (5-7), whereas autodephosphorylation is favored at night when KaiB binds to KaiC and sequesters KaiA $(8,9)$ (Fig. 1A). Although these three proteins alone can establish a circadian oscillation in vitro, two histidine protein kinases contribute to the complex in vivo: SasA and CikA, which coordinate a vast program of circadian gene expression executed by the response regulator, RpaA (10). The N-terminal thioredoxin-like domain of SasA is structurally homologous to a rare fold-switched monomer state of KaiB, and directly competes with this form of KaiB for binding to $\mathrm{KaiC}(11,12)$. This structural similarity and binding competition raised the possibility that SasA helps to regulate formation of the nighttime repressive complex, which is known to be restricted by $(i)$ the phosphorylation state of the KaiC CII domain (13), (ii) the intrinsically slow rate of ATP hydrolysis by the $\operatorname{KaiC} \mathrm{CI}$ domain $(14,15)$, and (iii) the rare conversion of KaiB to the its fold-switched form that is competent to bind KaiC (11). Similarly, CikA and KaiA bind to the same site on the KaiBC complex (9), and this competition shortens the period (11) and compensates for diminished concentrations of KaiA in the PTO in vitro (16), suggesting that output kinases fortify robustness of the PTO by modulating Kai protein interactions directly. 
To explore how the intrinsic structural similarity between fold-switched KaiB and the N-terminal thioredoxin-like domain of Sas A might influence the cyanobacterial PTO, we took advantage of a new high-throughput fluorescence polarization-based post-translational oscillator (FP-PTO) assay that reports on complex formation with $\mathrm{KaiC}$ in real time (17). This allowed us to capture circadian rhythms of quaternary complex formation with KaiC under standard assay conditions for the PTO (4) in the presence of $50 \mathrm{nM}$ fluorescently-labeled probes for KaiB or the thioredoxin-like domain of SasA, Sas $A_{\text {trx }}$, benchmarking the phase of these rhythms to the KaiC phosphorylation cycle (18) (Fig. 1B and S1). The phase of Sas $A_{\text {trx }}$ binding preceded that of KaiB by a few hours and was closer to the peak of KaiC phosphorylation (Fig. 1C), similar to that of full-length SasA (see accompanying paper by Chavan, A. et al.). Using KaiC phosphomimetics KaiC-EE and KaiC-EA, which are widely used to approximate the dusk-like pS,pT and nighttime-like pS,T states, we found that KaiB has similar affinity for both (Fig. S2). By contrast, full-length SasA has a higher preference for the earlier-occurring pS,pT state $(13,19)$. Although the N-terminal domain of SasA is necessary and sufficient for binding to KaiC (20), avidity effects in the full-length dimer enhanced affinity for KaiC-EE by at least two orders of magnitude compared to the isolated, monomeric Sas $A_{\text {trx }}$ domain (21) (Fig. S2). One other contribution to the delayed phase of KaiB-KaiC binding could be its population shift from a highly stable tetrameric KaiB ground state to an unstable monomeric fold (11).

Six monomers of the active, thioredoxin-like fold of KaiB assemble onto the KaiC hexamer of $S$. elongatus ( 8 ) and the related thermophilic species, T. elongatus (9) to nucleate formation of the nighttime repressive state (Fig. 1D). A high-resolution crystal structure of the sub-complex comprising a single KaiC-CI domain and a fold switch-locked mutant of KaiB I88A (I87A in $S$. 
elongatus, referred to herein as "fsKaiB" for both) illustrates how KaiB docks onto the exposed B-loop of the CI domain (Fig. 1E). We solved a crystal structure of the Sas $A_{\text {trx }}$ domain bound to the KaiC-CI domain from T. elongatus, revealing that SasA binds the B-loop in a similar orientation to KaiB (Fig. 1F and Table S3). To interrogate the importance of this interface, we probed several KaiC residues in the B-loop by mutagenesis and found that substitutions at sites conserved between S. elongatus and T. elongatus (F122 and D123) decrease affinity for both KaiB and SasA (Fig. 1G-I and S3). We then examined how differences in the structures of the thioredoxin-like domains of KaiB and SasA, or their orientations on the B-loop might influence binding to adjacent subunits of the KaiC hexamer (Fig. S3). Modest changes in the length and orientation of the C-terminal helix between the SasA and KaiB thioredoxin-like folds could lead to steric clashes of SasA with a neighboring subunit. Consistent with this idea, saturation binding experiments showed that $\mathrm{Sas}_{\mathrm{trx}}$ domains cannot fully occupy all six binding sites on the KaiC hexamer (Fig. S3). Moreover, unlike the highly cooperative binding observed for KaiB, SasA does not associate cooperatively with $\operatorname{KaiC}(21,22)$.

Prior studies of KaiB cooperativity demonstrated either one or six monomers of KaiB bound to the KaiC hexamer by native mass spectrometry $(21,22)$. Given the similarity between the SasAKaiC and KaiB-KaiC interactions, we wondered whether SasA could influence KaiB-KaiC interactions through heterotropic cooperativity. To test this idea, we performed equilibrium binding titrations of KaiC-EE with $50 \mathrm{nM}$ fluorescently-labeled KaiB probe in the absence or presence of unlabeled SasA or the fsKaiB as a secondary titrant (referred to herein as an 'additive' to the KaiC-EE titration, Fig. 2A, B). While we could not easily assess the degree of homotropic cooperativity for KaiC-EE with the $\mathrm{KaiB}$ probe alone under these assay conditions, 
low concentrations (e.g., 50-100 nM) of SasA or fsKaiB significantly increased binding of the probe, demonstrating more efficient recruitment of KaiB to the KaiC hexamer. Higher concentrations of additives competed with the probe to delay its binding to the KaiC hexamer until the equivalence point where the concentration of KaiC-EE matches the additive. at this point, indicative of positive heterotropic cooperativity (Fig. 2A, B). Binding of KaiB to the KaiC-CI monomer was not influenced by the addition of SasA or fsKaiB (Fig. S4), nor did Sas A interact directly with $\mathrm{KaiB}$ in the absence of KaiC (Fig. S4), suggesting that these thioredoxin-like folds enhance the cooperative enhancement of KaiB only on the KaiC hexamer. While cooperativity operates at the KaiC hexamer level, a simplified two-site thermodynamic model was sufficient to account for the data using least-squares fitting. (Fig. 2C and S4). We defined the heterotropic 'cooperativity index' as the fold-increase in KaiB affinity given by the ratio of equilibrium constants $\mathrm{K}_{1} / \mathrm{K}_{3}\left(=\mathrm{K}_{2} / \mathrm{K}_{4}\right)$. Comparison of two-dimensional (2D) titration assays with SasA or fsKaiB to simulated data representing heterotropic or homotropic cooperative binding, respectively (Fig. 2D), to a model with no cooperativity (Fig. 2E), suggests that SasA and fsKaiB similarly influence cooperative recruitment of the KaiB probe on KaiC.

To explore the role of intersubunit interactions in KaiB cooperativity, we first turned to the KaiB-R22A mutant originally identified in Anabaena (23), which reduces the apparent affinity of KaiB for KaiC. This residue has been implicated in KaiB cooperativity (21) and is situated at the KaiB-KaiB interface in the KaiBC hexamer structure (9). When this mutant was tested in our cooperativity assay, we observed a decrease in the cooperative recruitment of the KaiB probe relative to fsKaiB (Fig. 2G and S4), demonstrating that inter-subunit KaiB-KaiB interactions 
play a role in this cooperative recruitment process. Moreover, although SasA kinase activity is required for its role in regulating circadian rhythms of transcriptional output in vivo (20), we found that the kinase-dead SasA mutant H161A is as effective as wild-type SasA in stimulating cooperative recruitment of $\mathrm{KaiB}$ to $\mathrm{KaiC}$ in vitro (Fig. 2G).

To see whether SasA's role as a positive regulator of KaiBC association is important for its function in vivo, we set out to identify point mutations at potential cooperativity interfaces. We used crystal structures of Sas $\mathrm{A}_{\text {trx }}-\mathrm{CI}$ and fsKaiB-C1 to model a Sas $\mathrm{A}_{\text {trx }}-\mathrm{KaiC}-\mathrm{KaiB}$ complex to examine potential Sas $\mathrm{A}_{\text {trx }}-\mathrm{KaiB}$ interactions along the adjacent clockwise $(\mathrm{CW})$ and counterclockwise $(\mathrm{CCW})$ interfaces of the hexamer (Fig. 3A). Within this framework, we investigated a number of Sas A mutations near the $\mathrm{CW}$ and $\mathrm{CCW}$ interface to see how they would influence cooperative recruitment in KaiB binding assays (Fig. S5). Using analysis of 2D titration data with the mutants, we found a decrease in the cooperativity indices for the individual mutants H28A (CCW interface) or Q94A (CW interface) that was further decreased in the H28A/Q94A double mutant (Fig. 3B). The H28A/Q94A displayed a striking lack of heterocooperativity in the 2D titration assay (Fig. 3C compared to simulated data in $\mathbf{2 E}$ ), demonstrating that SasA-KaiB interactions at both interfaces are important for cooperative recruitment.

To see if the SasA-induced heterocooperativity we observed in vitro influences circadian rhythms in vivo, we introduced these substitutions into SasA using CRISPR/Cas12a and monitored bioluminescence from a PkaiBC luciferase reporter in constant light (LL) after synchronization in 12-h light:12-h dark cycles (Fig. 3D and S6). Modest effects on amplitude 
were seen with the individual H28 or Q94 alanine substitutions on their own, but when these were combined in the H28A/Q94A mutant, the amplitude of circadian rhythms was decreased to an extent that was similar to the SasA knockout strain ( $\Delta s a s A$, Fig. 3E). Furthermore, we observed a 2-h increase in period in the double-mutant strain (Fig. 3F), suggesting that heterocooperative interactions at the $\mathrm{CW}$ and $\mathrm{CCW}$ interfaces are redundant, but crucial for circadian timekeeping in vivo.

SasA was originally described as an amplifier of circadian rhythms, critical for maintaining robust rhythmicity by controlling rhythmic transcription of the kaiBC cluster (20). To test whether this amplitude-enhancing effect might be due in part to SasA regulation of KaiB binding to $\mathrm{KaiC}$, we turned to the FP-PTO assay to explore the effects of SasA on robustness of the core circadian oscillator in the absence of transcription-translation feedback that occurs in vivo (1). First, we measured the dependency of this oscillator assay on the concentrations of KaiA and KaiB, comparing data from the FP-PTO to a prior study using rhythms of KaiC phosphorylation to find a striking convergence in our results (Fig. 4A, B) (24). Because the FP-PTO is monitored non-invasively in 384-well format, we could follow the oscillator under different conditions for many days longer than is practical for the KaiC phosphorylation assay that is analyzed by SDSPAGE. Generally, oscillator amplitude and period were highly sensitive to KaiA concentrations, and while there was a clear requirement for a minimal concentration of KaiB, the period was quite robust to excess $\mathrm{KaiB}$ relative to $\mathrm{KaiC}$ (Fig. 4C-E). Although the oscillator functioned at low amplitude for several days with a normal circadian period with one-half stoichiometry of KaiB relative to KaiC (i.e., $1.75 \mu \mathrm{M}$ KaiB for $3.5 \mu \mathrm{M}$ KaiC) (24), these rhythms rapidly damped (Fig. 4B). KaiB is present in only modest excess over KaiC in vivo $(25,26)$, which suggests that 
the ability of KaiC to cooperatively recruit and bind six KaiB monomers is critical to sequester KaiA stably during negative feedback in the PTO and is thus a determinant of clock robustness. Consistent with this idea, the amplitude of the PTO decreased as the concentration of KaiA approached or exceeded that of KaiC (Fig. 4A, C and (13, 24)).

Because of its ability to enhance formation of the KaiBC complex, SasA has the potential to improve robustness of the PTO under limiting concentrations of KaiB by recruiting a stable, stoichiometric complement of KaiB to KaiC hexamers to sequester KaiA. To explore how SasA influences KaiB binding in the context of an oscillator, we set up FP-PTO assays under typical in vitro oscillator conditions (4) as well as under limiting concentrations of KaiB relative to KaiC. Low anisotropy levels for labeled KaiB under the limiting condition of only $0.875 \mu \mathrm{M}$ KaiB (Fig. 5A, left column) demonstrated incomplete KaiB binding to the KaiC hexamer. The addition of full-length SasA up to $1 \mu \mathrm{M}$ increased KaiB anisotropy levels, demonstrating that SasA enhanced $\mathrm{KaiB}$ binding to $\mathrm{KaiC}$ in the presence of KaiA, even without a functional oscillator. However, as the concentration of SasA approached or exceeded that of KaiC, anisotropy values for KaiB dropped, indicating that SasA outcompeted KaiB for binding to the KaiC hexamer as observed before $(12,27,28)$. When KaiB was present at one-half stoichiometry with respect to KaiC (1.75 $\mu \mathrm{M}$ KaiB for $3.5 \mu \mathrm{M}$ KaiC), the addition of SasA restored robust oscillations to the FP-PTO (Fig. 5A, middle column). This enhancement appears to be almost catalytic, as only 100 nM SasA was sufficient to rescue damping (Fig. 5B) and restore amplitude (Fig. 5C) to wildtype levels. Increasing Sas A concentrations to $1 \mu \mathrm{M}$ under these conditions did not influence amplitude any further, while higher concentrations of SasA outcompeted KaiB binding to attenuate oscillations in the FP-PTO (Fig. 5C). 
Under standard oscillator conditions with stoichiometric KaiB and KaiC, the presence of SasA up to $0.65 \mu \mathrm{M}$ did not markedly influence the oscillator (Fig. 5A, right column), whereas at increased concentrations of SasA, oscillator amplitude diminished until KaiB was completely outcompeted for KaiC binding (Fig. 5B, D). This suggests that the competitive effects of SasA may be enhanced as the concentration of KaiB equals KaiC. Moreover, the sharp drop in amplitude of the FP-PTO suggests that the functional switch from cooperativity to competition occurs around $\sim 1 \mu \mathrm{M}$ SasA depending on the exact oscillator conditions; interestingly, this ultrasensitive setpoint is close to the concentration of SasA in vivo estimated by quantitative western blotting $(0.58 \pm 0.07 \mu \mathrm{M}$ from (29)). In all cases where conditions were sufficient to generate a stable PTO, the period was lengthened similarly by addition of SasA (Fig. 5E). Therefore, in addition to regulating rhythms of transcriptional output from the Kai-based PTO (20), SasA also works to directly modulate KaiB association with KaiC to control formation of the nighttime repressive state and robustness of the PTO itself.

The other circadian output kinase, CikA, also contributes directly to robustness of the PTO by enhancing rhythms under limiting concentrations of KaiA (10). Using the FP-PTO assay, we observed that addition of CikA moderately enhanced low amplitude rhythms under limiting concentrations of KaiA while shortening the period (Fig. S7). The pseudo-receiver (PsR) domain of CikA binds to the same site on KaiB in the nighttime complex as KaiA does (9), and their competition in vivo is made evident by the phenotypes of a kaiA deletion mutant (30). By competing KaiA out of the repressive complex, CikA promotes the activating potential of KaiA to stimulate KaiC phosphorylation $(6,16)$. Either the isolated PsR domain or full-length CikA 
caused similar concentration-dependent decreases in period length in the FP-PTO assay, while the isolated thioredoxin-like domain of SasA was much less effective at lengthening the period compared to full-length SasA, likely due to avidity effects (Fig. S2 and S8).

The FP-PTO assay implemented here has uncovered roles for the output kinases as accessory components of the oscillator, expanding its functionality beyond the narrow concentrations and ratios that are tolerated in the traditional in vitro oscillator (24). This extended oscillator reveals how the clock maintains consistency in vivo throughout rhythmic changes in oscillator components that occur as part of transcription-translation feedback $(26,31)$ and protein turnover $(32,33)$, as well as providing an experimental platform for integrating the oscillator with the upstream and downstream components with which it interacts (see manuscript by Chavan, A. et al.).

\section{References and Notes:}

1. M. Ishiura et al., Expression of a gene cluster kaiABC as a circadian feedback process in cyanobacteria. Science 281, 1519-1523 (1998).

2. H. Iwasaki, T. Nishiwaki, Y. Kitayama, M. Nakajima, T. Kondo, KaiA-stimulated KaiC phosphorylation in circadian timing loops in cyanobacteria. Proc Natl Acad Sci U S A 99, 15788-15793 (2002).

3. J. Tomita, M. Nakajima, T. Kondo, H. Iwasaki, No transcription-translation feedback in circadian rhythm of KaiC phosphorylation. Science 307, 251-254 (2005).

4. M. Nakajima et al., Reconstitution of circadian oscillation of cyanobacterial KaiC phosphorylation in vitro. Science 308, 414-415 (2005).

5. Y. I. Kim, G. Dong, C. W. Carruthers, Jr., S. S. Golden, A. LiWang, The day/night switch in KaiC, a central oscillator component of the circadian clock of cyanobacteria. Proc Natl Acad Sci U S A 105, 12825-12830 (2008).

6. T. Nishiwaki-Ohkawa, Y. Kitayama, E. Ochiai, T. Kondo, Exchange of ADP with ATP in the CII ATPase domain promotes autophosphorylation of cyanobacterial clock protein KaiC. Proc Natl Acad Sci U S A 111, 4455-4460 (2014). Clock: Structure of KaiA Dimer in Complex with C-Terminal KaiC Peptides at 2.8 A Resolution. Biochemistry 54, 4575-4578 (2015). 
8. J. Snijder et al., Structures of the cyanobacterial circadian oscillator frozen in a fully assembled state. Science 355, 1181-1184 (2017).

9. R. Tseng et al., Structural basis of the day-night transition in a bacterial circadian clock. Science 355, 1174-1180 (2017).

10. J. S. Markson, J. R. Piechura, A. M. Puszynska, E. K. O'Shea, Circadian control of global gene expression by the cyanobacterial master regulator RpaA. Cell 155, 1396-1408 (2013).

11. Y. G. Chang et al., Circadian rhythms. A protein fold switch joins the circadian oscillator to clock output in cyanobacteria. Science 349, 324-328 (2015).

12. R. Tseng et al., Cooperative KaiA-KaiB-KaiC interactions affect KaiB/SasA competition in the circadian clock of cyanobacteria. J Mol Biol 426, 389-402 (2014).

13. J. Lin, J. Chew, U. Chockanathan, M. J. Rust, Mixtures of opposing phosphorylations within hexamers precisely time feedback in the cyanobacterial circadian clock. Proc Natl Acad Sci U S A 111, E3937-3945 (2014).

14. J. Abe et al., Circadian rhythms. Atomic-scale origins of slowness in the cyanobacterial circadian clock. Science 349, 312-316 (2015).

15. C. Phong, J. S. Markson, C. M. Wilhoite, M. J. Rust, Robust and tunable circadian rhythms from differentially sensitive catalytic domains. Proc Natl Acad Sci U S A 110, 1124-1129 (2013).

16. M. Kaur, A. Ng, P. Kim, C. Diekman, Y. I. Kim, CikA Modulates the Effect of KaiA on the Period of the Circadian Oscillation in KaiC Phosphorylation. J Biol Rhythms 34, 218223 (2019).

17. J. Heisler, A. Chavan, Y. G. Chang, A. LiWang, Real-Time In Vitro Fluorescence Anisotropy of the Cyanobacterial Circadian Clock. Methods Protoc 2, (2019).

18. M. J. Rust, J. S. Markson, W. S. Lane, D. S. Fisher, E. K. O'Shea, Ordered phosphorylation governs oscillation of a three-protein circadian clock. Science 318, 809812 (2007).

19. S. J. Valencia et al., Phase-dependent generation and transmission of time information by the KaiABC circadian clock oscillator through SasA-KaiC interaction in cyanobacteria. Genes Cells 17, 398-419 (2012).

20. H. Iwasaki et al., A kaiC-interacting sensory histidine kinase, SasA, necessary to sustain robust circadian oscillation in cyanobacteria. Cell 101, 223-233 (2000).

21. R. Murakami et al., Cooperative Binding of KaiB to the KaiC Hexamer Ensures Accurate Circadian Clock Oscillation in Cyanobacteria. Int J Mol Sci 20, (2019).

22. J. Snijder et al., Insight into cyanobacterial circadian timing from structural details of the KaiB-KaiC interaction. Proc Natl Acad Sci U S A 111, 1379-1384 (2014).

23. R. G. Garces, N. Wu, W. Gillon, E. F. Pai, Anabaena circadian clock proteins KaiA and KaiB reveal a potential common binding site to their partner KaiC. EMBO J 23, 16881698 (2004).

24. M. Nakajima, H. Ito, T. Kondo, In vitro regulation of circadian phosphorylation rhythm of cyanobacterial clock protein KaiC by KaiA and KaiB. FEBS Lett 584, 898-902 (2010).

25. J. Chew, E. Leypunskiy, J. Lin, A. Murugan, M. J. Rust, High protein copy number is required to suppress stochasticity in the cyanobacterial circadian clock. Nat Commun 9 , 3004 (2018). 
26. Y. Kitayama, H. Iwasaki, T. Nishiwaki, T. Kondo, KaiB functions as an attenuator of KaiC phosphorylation in the cyanobacterial circadian clock system. EMBO J 22, 21272134 (2003).

27. A. H. Chen, D. Lubkowicz, V. Yeong, R. L. Chang, P. A. Silver, Transplantability of a circadian clock to a noncircadian organism. Sci Adv 1, (2015).

28. R. Murakami et al., The roles of the dimeric and tetrameric structures of the clock protein $\mathrm{KaiB}$ in the generation of circadian oscillations in cyanobacteria. J Biol Chem 287, 29506-29515 (2012).

29. A. Gutu, E. K. O'Shea, Two antagonistic clock-regulated histidine kinases time the activation of circadian gene expression. Mol Cell 50, 288-294 (2013).

30. D. G. Welkie et al., Genome-wide fitness assessment during diurnal growth reveals an expanded role of the cyanobacterial circadian clock protein KaiA. Proc Natl Acad Sci U $S$ A 115, E7174-E7183 (2018).

31. Y. Xu, T. Mori, C. H. Johnson, Circadian clock-protein expression in cyanobacteria:

32. C. K. Holtman et al., High-throughput functional analysis of the Synechococcus elongatus PCC 7942 genome. DNA Res 12, 103-115 (2005).

33. K. Imai, Y. Kitayama, T. Kondo, Elucidation of the role of clp protease components in circadian rhythm by genetic deletion and overexpression in cyanobacteria. J Bacteriol 195, 4517-4526 (2013).

\section{Acknowledgments:}

We thank staff at the 23-ID-D beamline of the Advanced Photon Source, Argonne National Laboratory for their help with data collection. The Advanced Photon Source (contract DE-AC02-

performed with UCSF Chimera and ChimeraX, developed by the Resource for Biocomputing,

Visualization, and Informatics at the University of California, San Francisco, with support from National Institutes of Health P41-GM103311(Chimera) and R01-GM129325 and the Office of Cyber Infrastructure and Computational Biology, National Institute of Allergy and Infectious

Diseases (ChimeraX). We thank Archana G. Chavan, Michael Rust, Lu Hong, Shahar Sukenik, Maria Zoghbi for useful discussions.

\section{Funding:}


This work was supported by National Institutes of Health grants R01 R35 GM118290 (to

S.S.G.), R01 GM121507 (to C.L.P.), and R01 GM107521 (to A.L.), and Department of the Army Research Office grant W911NF-17-1-0434 (to A.L.). J.H. was supported by the NSF-

CREST CCBM HRD-1547848. J.P. was supported by NIH IMSD grant R25 GM058903-20.

P.C. is supported by EMBO long-term fellowship 57-2019.

\section{Author contributions:}

Conceptualization, J.H., J.A.S., C.L.P., and A.L.; Methodology, J.H., J.A.S., and C.R.B.;

Investigation, J.H., J.A.S., J.G.P., C.S., D.C.E., R.K.S., and S.T.; Validation, J.H., J.A.S., and

S.T.; Formal Analysis, J.H., J.A.S., C.R.B., and P.C.; Resources, S.S.G; Data Curation, J.H. and

J.A.S.; Writing - Original Draft, J.H., J.A.S., and C.L.P.; Writing - Reviewing and Editing, J.H.,

J.A.S., D.C.E., C.R.B., S.S.G., C.L.P., and A.L.; Funding Acquisition, J.H., J.P., P.C., S.S.G.,

C.L.P., and A.L.; Supervision, S.S.G., C.L.P., and A.L.

Competing interests: Authors declare no competing interests.

Data and materials availability: All data is available in the main text or Supplementary

Materials.

\section{Supplementary Materials:}

Materials and Methods

Figures S1-S8

Tables S1-S10

References (1-27) 


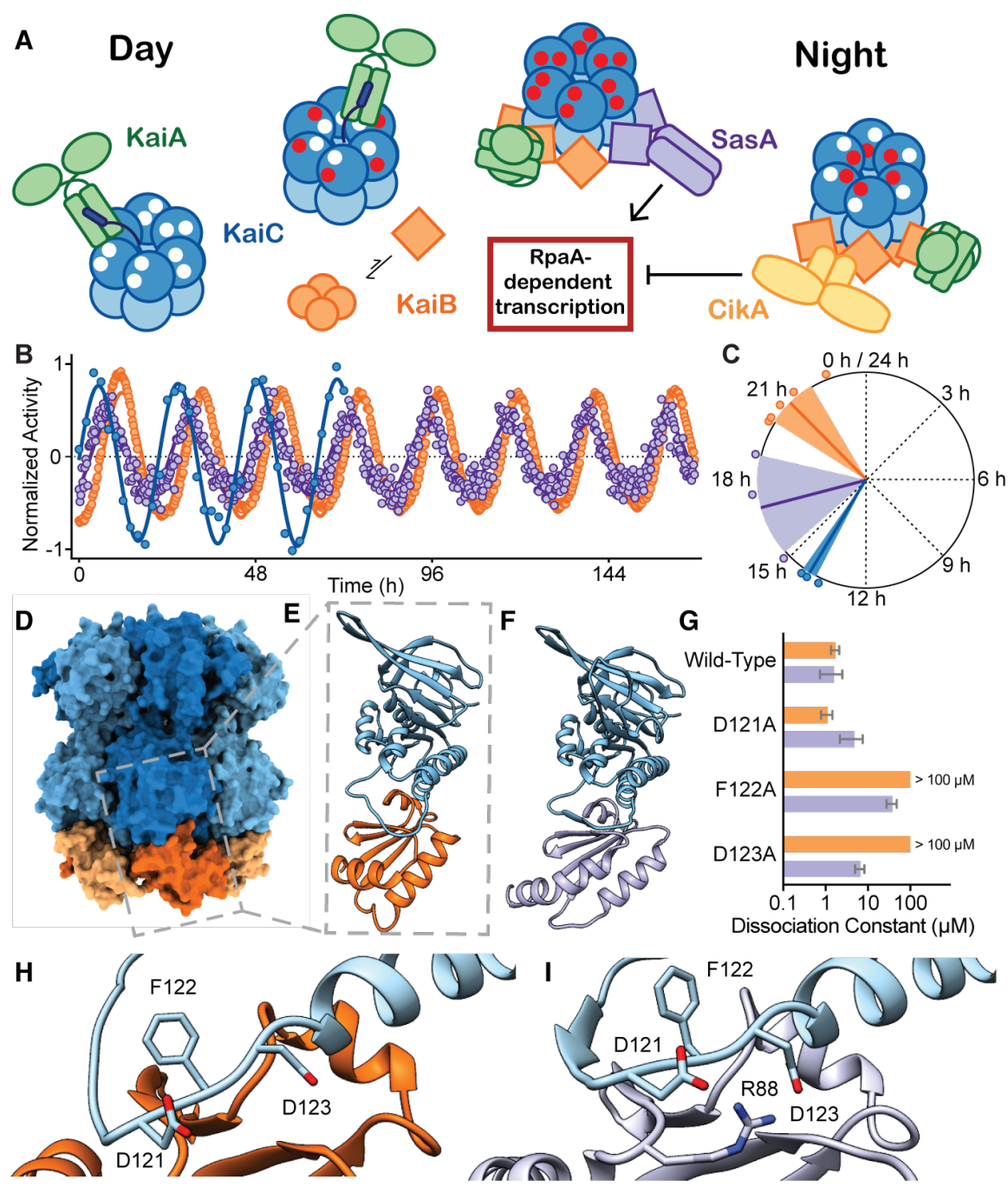

Figure 1: SasA and KaiB bind with sequential but overlapping phases to the same site on

KaiC. A) Cartoon schematic of the cyanobacterial oscillator and associated output pathways.

The KaiC phosphorylation rhythm (18) is depicted with white (unphosphorylated) or red

(phosphorylated) circles. Ground-state KaiB tetramer (circles) interchanges with the fold-

switched thioredoxin-like fold (squares). B) Normalized rhythms of KaiC phosphorylation (blue, measured by SDS-PAGE for $72 \mathrm{~h}$ ) with KaiB or Sas $\mathrm{A}_{\text {trx }}$ association with KaiC (orange or purple, respectively). Data points from individual traces $(n=3)$ are overlaid in circles, and the mean (solid line) fitted to a sine function. See Fig. S1 for raw data. C) Rayleigh phase diagram of KaiC phosphorylation and $\mathrm{KaiB}$ or $\mathrm{Sas}_{\mathrm{trx}}$ binding for in vitro oscillations. Dark line, mean 
phase of KaiC binding or phosphorylation (circles represent $n \geq 3$ assays; SD, width of the light wedge). Calculated phases are KaiB: $20.9 \pm 0.9$ h, SasA: $17.1 \pm 1.7$ h, and KaiC-P: $14.0 \pm 0.4$ h. D) The KaiB-KaiC hexamer (PDB:5JWQ). Subunits of KaiC are depicted in alternating light/dark blue, and fsKaiB monomers alternate yellow/orange. Gray box, position of the KaiCCI domain bound to KaiB. E) The KaiC-CI domain-fsKaiB subcomplex (PDB:5JWO), with KaiC in blue, KaiB in orange. F) The KaiC-CI domain-SasA $\mathrm{trx}_{\text {subcomplex }}$ (PDB:6X61), with KaiC in blue, KaiB in orange. G) Equilibrium dissociation constants $\left(\mathrm{K}_{\mathrm{D}}\right)$ of $\mathrm{KaiC}-\mathrm{EE}$ and mutants for KaiB or $\mathrm{Sas}_{\text {trx }}$ (mean $\pm \mathrm{SEM}, \mathrm{n}=3$ ). Where indicated, binding was too weak for curve-fitting and $\mathrm{K}_{\mathrm{D}}$ is reported at $>100 \mu \mathrm{M}$. H-I) The interface of KaiC-CI with fsKaiB (H) or $\operatorname{Sas}_{\text {trx }}(\mathbf{I})$ with key residues highlighted. 

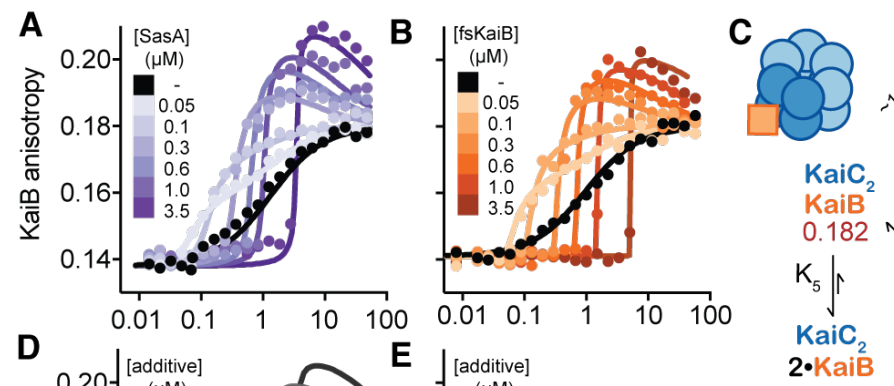

KaiB + SasA

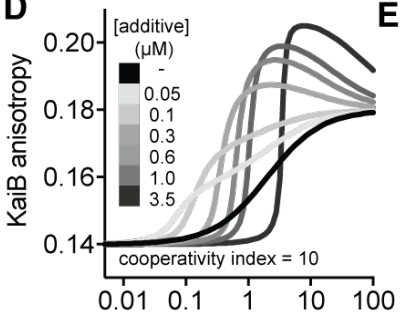

E [additive]
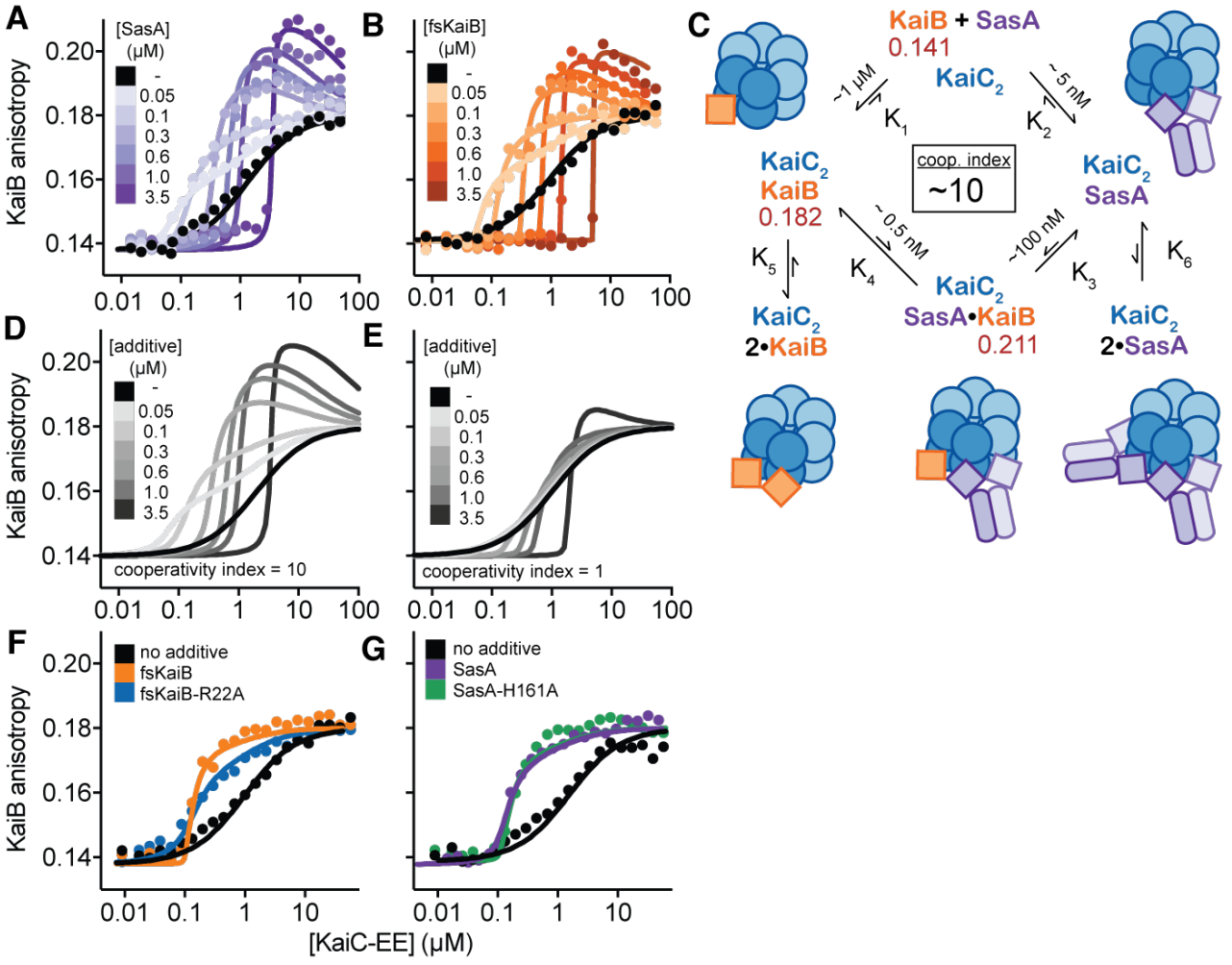

Figure 2: The thioredoxin-like fold of SasA and fsKaiB cooperatively recruits KaiB to the

KaiC hexamer. A-B) Titrations of fluorescently-labeled KaiB with KaiC-EE in the absence (black) or presence of unlabeled full-length SasA (A, light to dark purple) or fsKaiB (B, light to dark orange). 2D titrations are shown from representative assays with global fitting to the thermodynamic model (see Fig. S4). C) Summary of two-site thermodynamic model for SasA heterocooperativity (darker shades) with the relevant higher-order species depicted in lighter colors. Mean equilibrium constants derived from least squares fitting of SasA 2D titration assays $(\mathrm{n}=3)$ (Data S3); red, mean peak anisotropy values observed from global fitting of SasA 2D titration datasets. The cooperativity index is defined as the ratio $\mathrm{K}_{1} / \mathrm{K}_{3}=\mathrm{K}_{2} / \mathrm{K}_{4}$. D-E) Simulated 2D titration data from thermodynamic model with a cooperativity index $=10(\mathbf{D})$ or $1(\mathbf{E}) . \mathbf{F})$ Titrations of fluorescently-labeled KaiB with KaiC-EE in the presence of $100 \mathrm{nM}$ fsKaiB (orange) or fsKaiB-R22A (blue, full titration dataset in Fig. S4). G) Titrations of fluorescently- 
bioRxiv preprint doi: https://doi.org/10.1101/2020.06.17.158394; this version posted June 19, 2020. The copyright holder for this preprint (which was not certified by peer review) is the author/funder, who has granted bioRxiv a license to display the preprint in perpetuity. It is made available under aCC-BY-NC-ND 4.0 International license.

labeled KaiB with KaiC-EE in the presence of $100 \mathrm{nM}$ SasA (purple) or SasA-H161 A (green, full titration dataset in Fig. S4). 

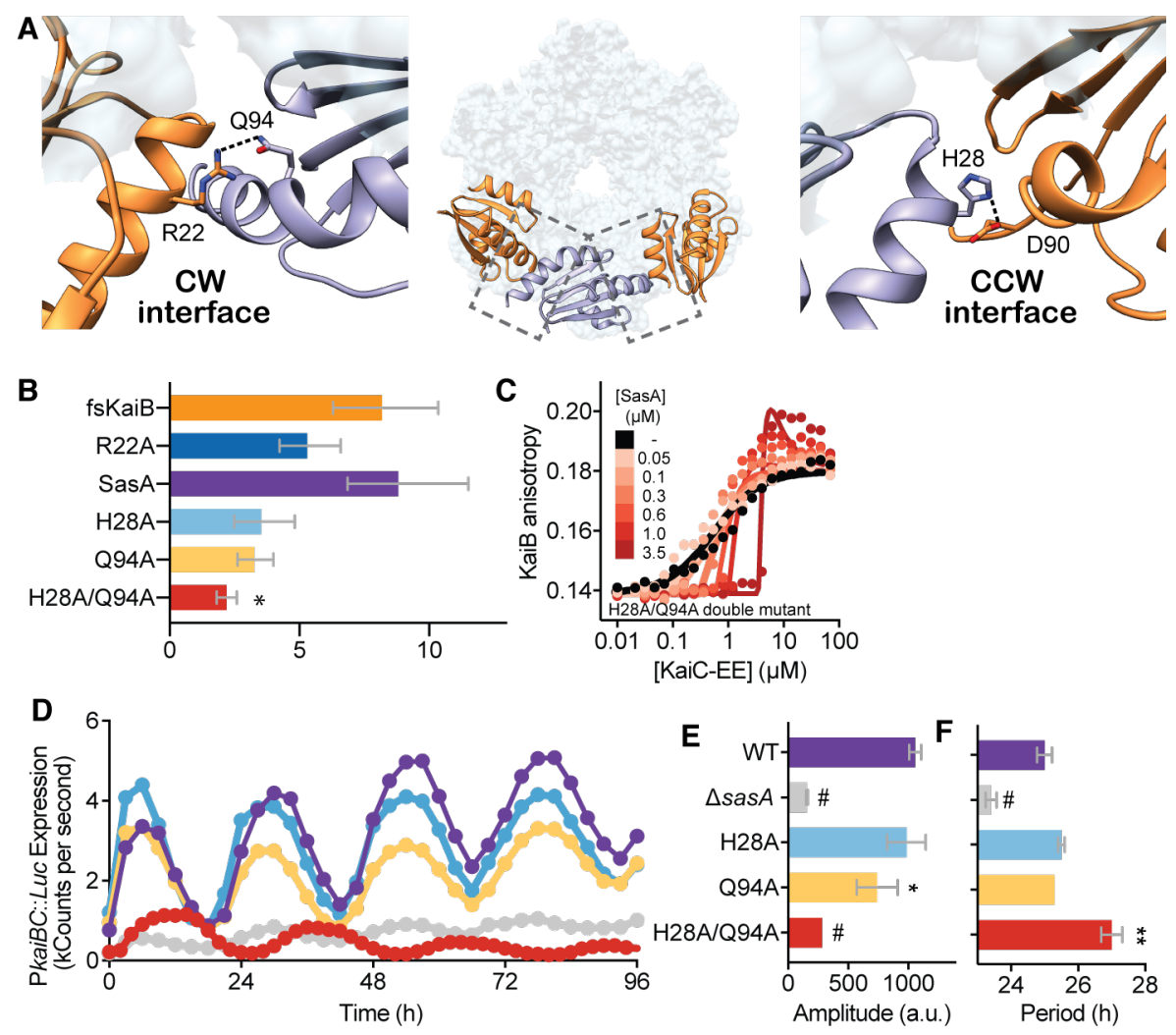

Figure 3: SasA-KaiB interactions mediate cooperative recruitment of KaiB in vitro and sustain robust circadian rhythms in vivo. A) Structural model of KaiB:SasA interactions on KaiC from KaiC-CI subcomplexes with fsKaiB (orange, PDB:5JWO) or Sas $\mathrm{A}_{\text {trx }}$ (purple, PDB:6X61) modeled onto adjacent subunits of KaiC (light blue) of the KaiBC hexamer (PDB:5JWQ). Key residues at clockwise (CW) and counterclockwise (CCW) interfaces are modeled as S. elongatus variants based on the alignment in Fig. S5. Dotted lines, polar contacts predicted by hybrid structural model. B) Cooperativity indices for KaiB and SasA mutants from thermodynamic model. Bars represent median and 95\% confidence intervals from Monte Carlo analysis from $n=2-3$ full 2D datasets. See Supplemental Methods for more details. Statistical analysis was performed on variance between median Monte Carlo values between replicates (*, $P<0.05)$ C) Representative 2D titration of fluorescently-labeled KaiB with KaiC-EE in the absence (black) or presence of SasA-H28A/Q94A (light to dark red). D) Representative 
timecourse of bioluminescence driven by PkaiBC from S. elongatus cultures entrained under 12-

h LD cycles for 48 -hr and subsequently allowed to free run in LL. Raw luminescence curves

were fit to a sine function to extract amplitude $(\mathbf{E})$ and free running circadian period $(\mathbf{F})$ for each strain. Error bars depict standard deviation among replicate cultures $(n=6-12)$; when error bars are not visible, they were smaller than could be depicted. Symbols indicate significance $(* *, P<$ 0.01; \#, $P<0.0001)$ from one-way ANOVA of mutants relative to wild-type $S$. elongatus. 

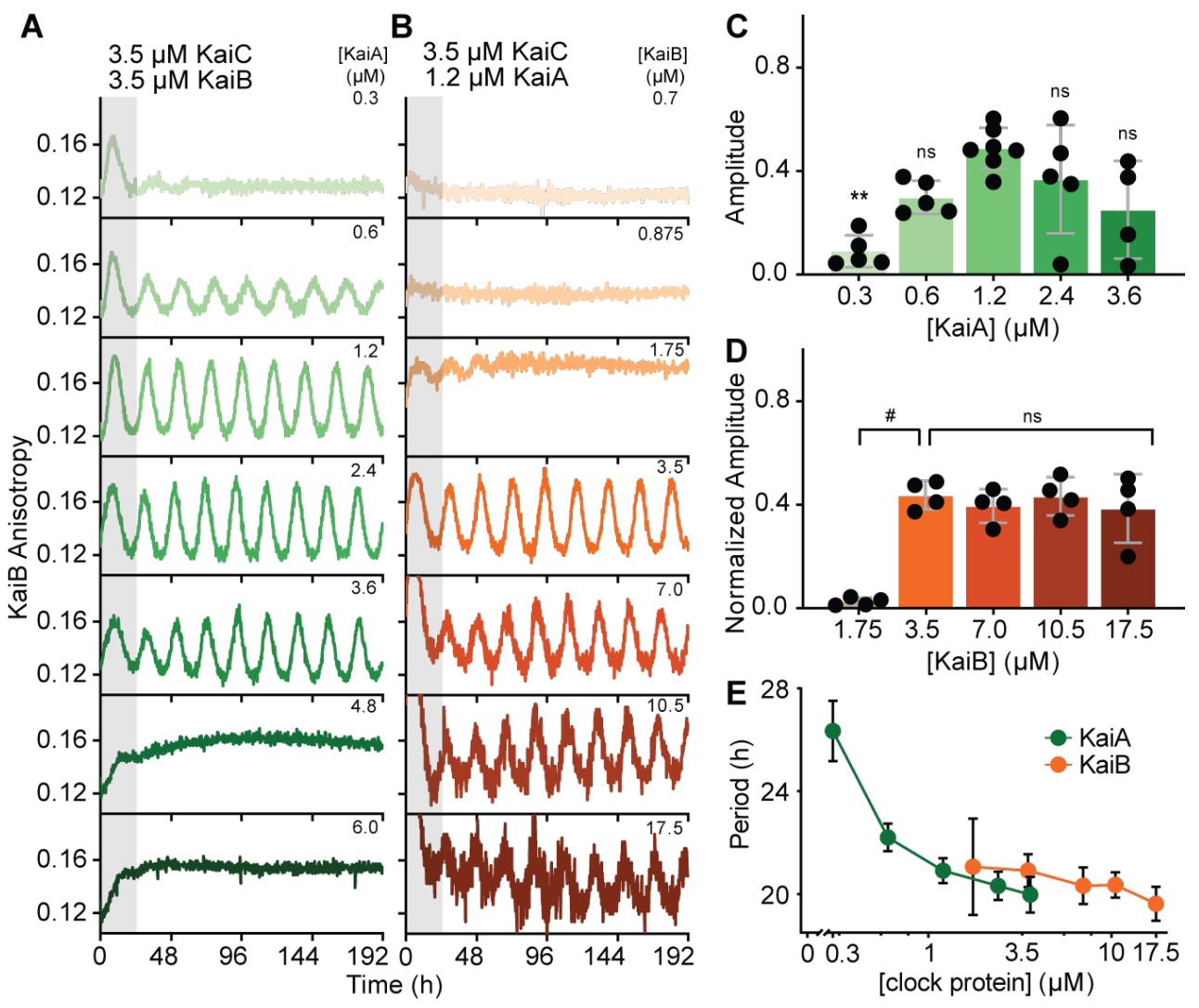

Figure 4: Amplitude and period of the oscillator depend in different ways on the

concentrations of KaiA and KaiB. A) The FP-PTO in vitro with standard conditions of $3.5 \mu \mathrm{M}$ KaiC, $3.5 \mu \mathrm{M}$ KaiB (including $50 \mathrm{nM}$ fluorescently-labeled KaiB as a probe) and titrations of

KaiA from 0.3-6.0 $\mu \mathrm{M}$. Representative assay from $\mathrm{n}=3$ shown; the first 24-hr period after

release into constant conditions is marked in gray. B) The FP-PTO with standard conditions of $3.5 \mu \mathrm{M}$ KaiC, $1.2 \mu \mathrm{M}$ KaiA and titrations of $\mathrm{KaiB}$ from 0.7-17.5 $\mu \mathrm{M}$ (including $50 \mathrm{nM}$ fluorescently-labeled KaiB as a probe). C-D) Amplitude analysis of KaiA titrations (C, data from panel A) or KaiB titrations (D, data from panel B). Amplitudes for KaiB titrations were normalized to account for increasing ratio of unlabeled KaiB to fluorescently-labeled KaiB. Reaction conditions that could not be fit by FFT-NLLS analysis, and therefore did not oscillate, were not included. Data are shown as mean $\pm \mathrm{SD}(\mathrm{n} \geq 3)$. One-way ANOVA with Dunnett's multiple comparisons test was used for comparison between groups: ns, non-significant; *, $P<$ 
bioRxiv preprint doi: https://doi.org/10.1101/2020.06.17.158394; this version posted June 19, 2020. The copyright holder for this preprint (which was not certified by peer review) is the author/funder, who has granted bioRxiv a license to display the preprint in perpetuity. It is made available under aCC-BY-NC-ND 4.0 International license.

$0.05 ; * *, P<0.01 ; * * *, P<0.001$; \#, $P<0.0001$. E) Period of the FP-PTO plotted against KaiA

(green) or KaiB (orange) concentrations. Data are shown as mean $\pm \mathrm{SD}(\mathrm{n} \geq 3)$. 


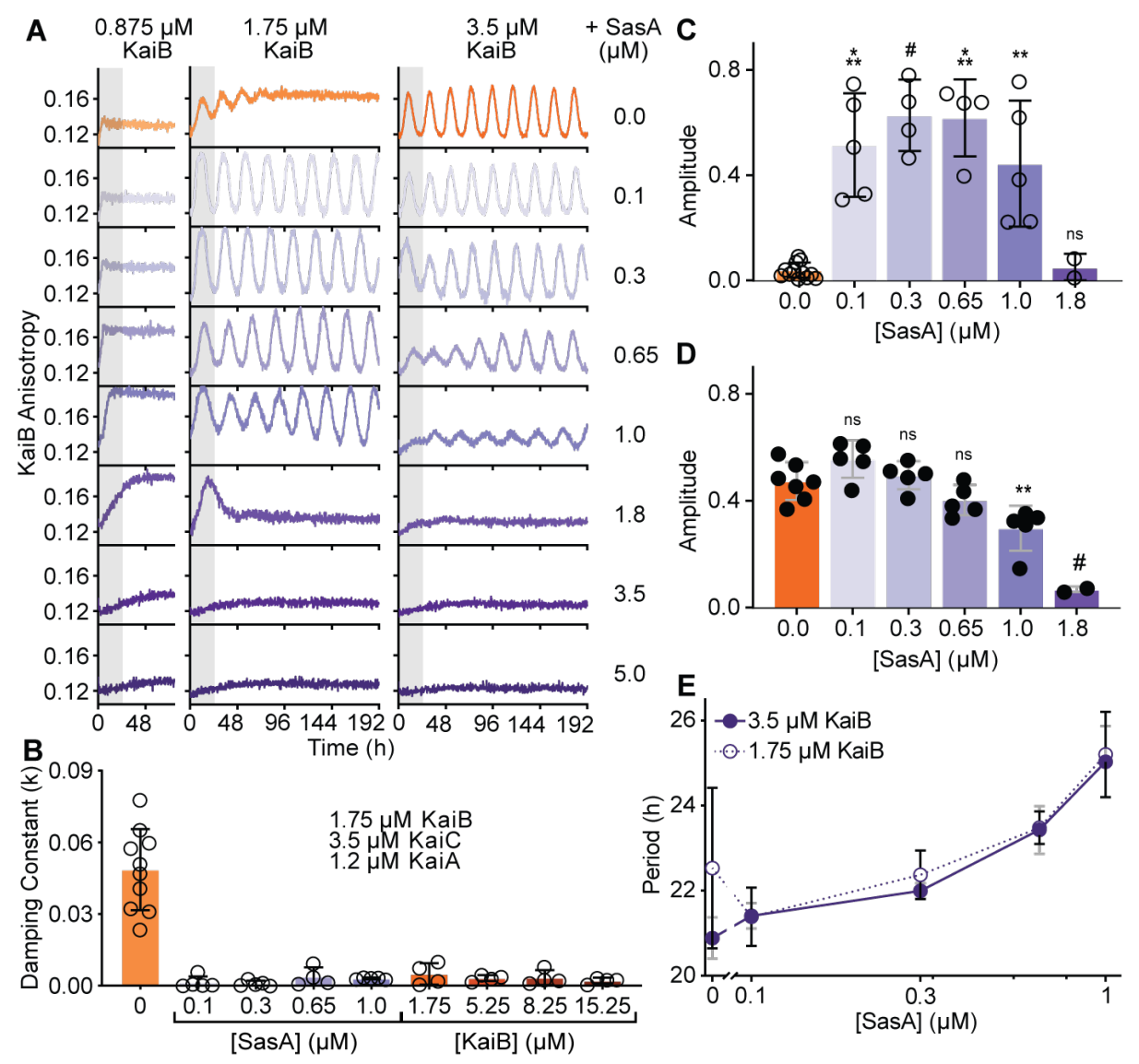

Figure 5: SasA dynamically influences robustness of the post-translational oscillator in

vitro. A) The FP-PTO with $3.5 \mu \mathrm{M}$ KaiC, $1.2 \mu \mathrm{M}$ KaiA, and the indicated concentrations of KaiB (including $50 \mathrm{nM}$ fluorescently-labeled KaiB as a probe) with titrations of full-length SasA constant conditions is marked in gray. B) Damping constants of the FP-PTO with $1.75 \mu \mathrm{M}$ KaiB (including $50 \mathrm{nM}$ fluorescently-labeled KaiB as a probe) in the presence of added SasA (purple) or KaiB (orange). C-D) Amplitude of the FP-PTO prepared with $1.75 \mu \mathrm{M} \mathrm{KaiB}(\mathbf{C}$, open circles) or $3.5 \mu \mathrm{M}$ KaiB (D, closed circles) in the presence of SasA (light to dark purple). E)

Period of the FP-PTO prepared with $1.75 \mu \mathrm{M}$ KaiB (open circles) or $3.5 \mu \mathrm{M}$ KaiB (closed circles) with SasA (light to dark purple). Reaction conditions that could not be fit by FFT-NLLS analysis, and therefore did not oscillate, were not included. Damping constant, period, and 
amplitude data are representative of four or more independent experiments, are presented individually and as the mean $\pm \mathrm{SD}$. One-way ANOVA with Dunnett's multiple comparison test was used for comparison between groups: ns, non-significant; *, $P<0.05$; **, $P<0.01$; **, $P<$ $0.001 ; \#, P<0.0001$. 